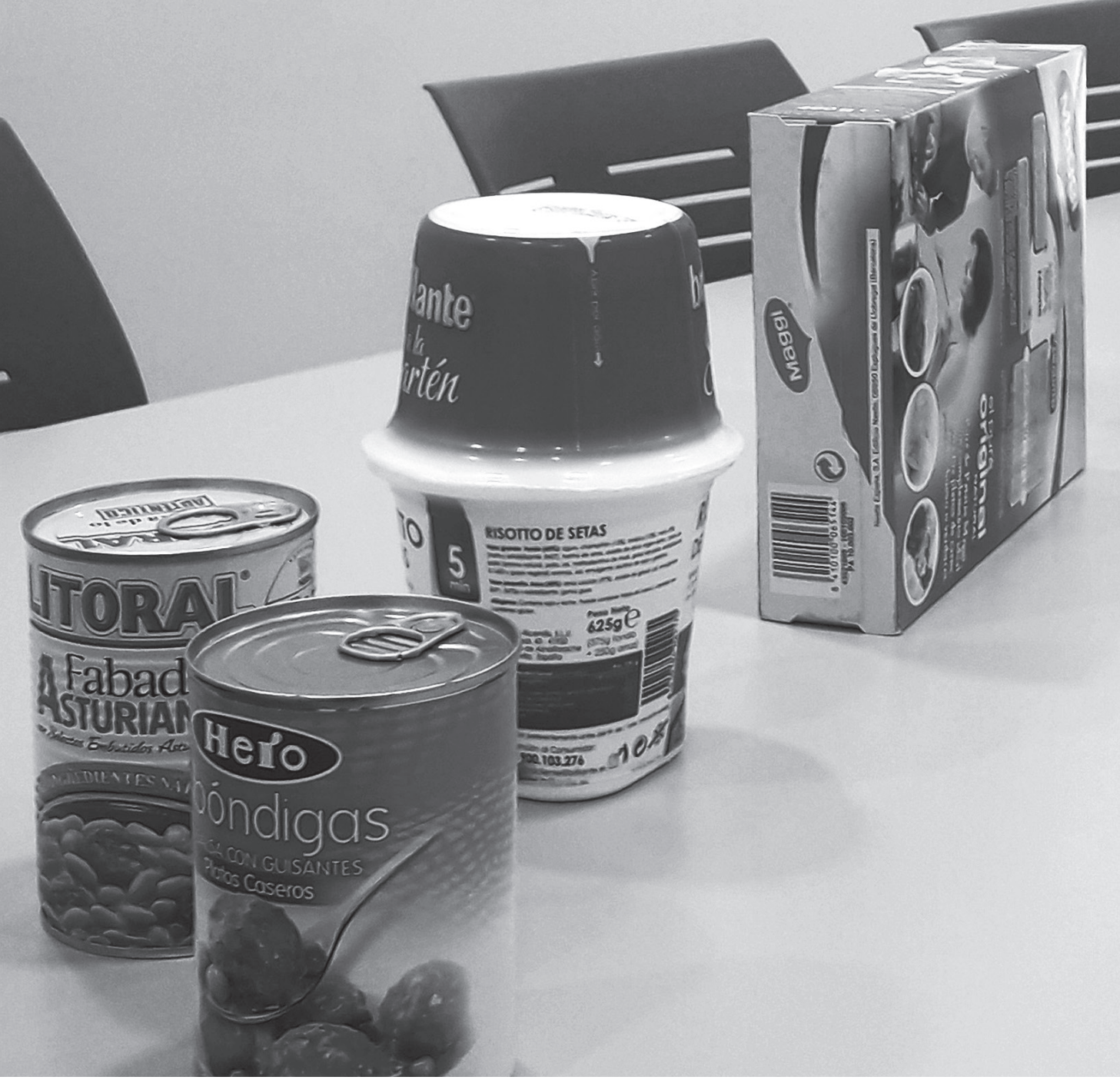

AIBR

Revista de Antropología

Iberoamericana

www.aibr.org

Volumen 14

Número 1

Enero - Abril 2019

Pp. 145 - 167

Madrid: Antropólogos

Iberoamericanos en Red.

ISSN: 1695-9752

E-ISSN: 1578-9705

\section{Maneras de clasificar los alimentos y elecciones alimentarias}

\section{Elena Espeitx Bernat}

Universidad de Zaragoza

Juanjo Cáceres Nevot

Universitat Oberta de Catalunya

Recibido: 16.01 .2018

Aceptado: 27.09 .2018

Dol: 10.11156/aibr.140108 


\section{RESUMEN}

En el presente artículo se presentan los resultados de un estudio realizado los años 2014-15 y desarrollado en Aragón y en Cataluña. El objetivo ha consistido en explorar las categorías a partir de las cuales se clasifican los alimentos en nuestra sociedad, como las relativas a la construcción del cuerpo. Para ello se ha utilizado una dinámica de grupo específica, diseñada para que dichas categorías surjan en la discusión de grupo, sin ser en ningún momento sugeridas por el/la moderador/a.

\section{PALABRAS CLAVE}

Alimentos saludables, natural, artificial, peligroso, nocivo.

\section{WAYS TO CLASSIFY FOOD AND FOOD CHOICES}

\section{ABSTRACT}

We present the results of a study conducted in the years 2014-15 and developed in Aragon and Catalonia (Spain). Our aim has been to explore the categories used in our society for classifying food. To this end, a specific focus group dynamic has been designed in order to allow the spontaneous emergence of the categories without being suggested at any time by the moderator.

KEY WORDS

Healthy foods, natural, artificial, dangerous, harmful. 


\section{Introducción: categorías de alimentos y cuerpo}

En las diferentes culturas y a lo largo del tiempo, la alimentación ha sido percibida como un elemento central en la construcción del cuerpo. La idea de que «somos lo que comemos» tiene hondas raíces y se refiere tanto a la construcción de nuestro cuerpo físico como a la construcción de nuestra identidad personal. Actualmente, la concepción de alimento como factor relevante en la construcción de cuerpo no solo sigue vigente, sino que ha cobrado una gran fuerza. Y esta estrecha relación percibida entre lo que ingerimos y la construcción del propio cuerpo se refleja en ciertas categorías mediante las cuales clasificamos los alimentos.

En todas las culturas encontramos formas específicas de categorizar los alimentos, entre las cuales la primera, universal e ineludible, es la que separa los seres vivos, animales o plantas, en dos grandes categorías: «comestible» $\mathrm{y}$ «no comestible». A partir de esta clasificación básica, cada sociedad establece un cierto número de categorías de alimentos, en función de diferentes criterios, como la situación de consumo (por ejemplo, cotidiano o festivo), las propiedades que se les atribuyen, su accesibilidad, su disponibilidad o su sabor (Contreras, 1993), y lo mismo sucede en nuestra sociedad. Antes de continuar, es preciso especificar a qué nos referimos al decir «nuestra sociedad». Las categorías generales que vamos a tratar son probablemente comunes a lo que llamamos "sociedad occidental», o como mínimo son extensibles al contexto europeo. Pero los contenidos de cada categoría pueden variar considerablemente de un territorio a otro. Dos grasas, la mantequilla y el aceite, serán categorizadas de manera distinta en Francia y en España. Así, aunque la categorización de los alimentos como «industriales», por ejemplo, puede ser ampliamente compartida, otros elementos presentan una mayor variabilidad según dónde nos situemos. Incluso en un mismo país pueden detectarse diferencias entre zonas urbanas y zonas rurales. Como «nuestra sociedad», pues, entendemos la España urbana en las primeras décadas del siglo XXI.

Ahora bien, incluso dentro de este espacio acotado, la inclusión de un alimento en una categoría u otra no es en absoluto homogénea. Un mismo alimento puede adscribirse a categorías distintas y casi siempre lo hace. Las valoraciones relativas a los alimentos son multidimensionales y cada una de estas dimensiones (palatibilidad, saludabilidad, familiaridad...) los ubica en categorías diferentes. Las categorías son también portadoras de valencias positivas y negativas: la valencia de la categoría «alimentos saludables» sería positiva, mientras que la de "alimentos contaminados» sería negativa. De ello se deriva que, dada la multidimensionalidad anteriormente citada, un mismo alimento puede adscribirse a 
una determinada categoría con valencia positiva y a otra categoría con valencia negativa. Por ejemplo, si pensamos un alimento desde la categoría "sabroso/no sabroso", la valencia puede ser muy positiva, mientras que, si lo pensamos desde la categoría «saludable/no saludable», esta puede ser muy negativa.

La percepción de que mediante la comida nuestro aspecto físico se modifica está en la base de las categorías relativas a la construcción del cuerpo. Según cuál sea nuestra forma de alimentarnos, nuestro pelo, nuestra piel, tendrán un mejor o peor aspecto. Pero, sobre todo, de lo que comemos dependerá nuestro peso (Gracia-Arnaiz, 2010). Delgadez, sobrepeso, obesidad, aparecen como indicadores potentes, cargados de múltiples significados. Son indicadores de estatus, existiendo, en nuestro contexto, una clara correlación inversa entre Índice de Masa Corporal y nivel de ingresos, lo que se ha dado en llamar el gradiente social de la obesidad (Robertson, Lobston y Knai, 2007). Son, asimismo, indicadores de aquello que llamamos «personalidad». Está bien documentada desde hace décadas la tendencia a atribuir cualidades negativas a las personas con un peso elevado, convirtiendo la obesidad en un rasgo corporal altamente estigmatizante. Estas características atribuidas en función del peso trascienden ampliamente el aspecto físico, definiendo de manera íntegra a la persona. Nos referimos a las asociaciones estrechas que se establecen entre obesidad y pereza, indolencia, incompetencia e incluso falta de inteligencia. O las que se establecen entre delgadez y dinamismo o eficiencia (Ajzen y Fishbein, 1980). Y es también, por supuesto, un indicador de atractivo sexual, o de ausencia de este.

La alimentación se relaciona también con otra dimensión corporal, la de la condición física. Lo que comemos influye en nuestra forma física $y$, aunque esta es percibida en primera instancia y sobre todo dependiente del ejercicio físico, también se atribuye un papel importante a la alimentación para alcanzarla, aunque estas atribuciones tengan, a menudo, escasa justificación (Basulto y Cáceres, 2014).

Tienen asimismo un gran peso las consideraciones relativas al mantenimiento de la salud y la prevención de la enfermedad. La concepción de que la salud es altamente tributaria de lo que se come no es nueva ni exclusiva de nuestro contexto sociohistórico, muy al contrario (Contreras, 1993), pero actualmente los discursos y las recomendaciones al respecto alcanzan una enorme resonancia. Más allá de la prevención de la enfermedad, también se perciben estrechas las relaciones entre lo que comemos y el bienestar corporal, especialmente en cuanto a cómo nos sentimos y vivimos diariamente en nuestro cuerpo. El tránsito intestinal, el nivel de energía, las digestiones difíciles, la flatulencia, las náuseas, la acidez o la 
sensación de cansancio son estados corporales percibidos como tributarios de la alimentación.

Finalmente, nuestro bienestar corporal y nuestra salud son considerados también dependientes de otra dimensión de los alimentos: su potencial toxicidad. Un alimento puede enfermarnos, incluso matarnos. Esta dimensión, al estar asociada a la supervivencia misma, explica la rápida difusión e intenso calado de las atribuciones de contaminación a los alimentos. La noción de riesgo se asocia a la ingesta alimentaria de manera robusta (Bredahl, Grunert y Frewer, 1998; Contreras, 2005; Costa Font, Gil, Trail y Bruce, 2008; Lusk, Jamal, Kurlander, Roucan y Taulman, 2005). Todas las asociaciones que hemos citado hasta ahora, vinculadas a nuestra percepción de las interacciones entre lo que comemos y cómo es y cómo experimentamos nuestro cuerpo, se despliegan en categorías como las de «alimento saludable», «engordante», "seguro» o "tóxico», entre otras.

Por supuesto, no todas las categorías se refieren a la construcción del cuerpo. Los alimentos se categorizan también como «ordinarios» — de consumo habitual—o «extraordinarios» — propios de situaciones especiales-, o como «familiares» o «exóticos», por ejemplo. Pero no todas las categorías tienen el mismo peso y presencia. Algunas, como las relativas a las dimensiones éticas o políticas de los alimentos, pueden ser relevantes para algunas personas y totalmente irrelevantes para otras. Otras categorías son omnipresentes, como la de los «alimentos que gustan/disgustan».

Ahora bien, aunque algunas pueden parecer, en primera instancia, ampliamente compartidas ( «alimentos saludables» o «alimentos festivos», por ejemplo), es sabido que existe una notable disparidad respecto a los alimentos que los individuos incluyen en cada una de estas categorías (Basulto y Cáceres, 2016). La bollería industrial es categorizada mayoritariamente como "poco saludable», pero para muchos las galletas no son bollería. Y aunque es altamente improbable que se incluya la bollería industrial en la categoría de "alimentos saludables», o un plato de acelgas cocidas en la categoría de "alimentos festivos», existe un gran número de productos y platos con fronteras mucho menos nítidas y que son clasificados de formas muy distintas.

En realidad, parece ser mayor el número de alimentos ambiguos que el de alimentos que pertenezcan claramente, de manera prácticamente unánime, a una determinada categoría, y que se mantengan de manera estable en ella. Tomemos, por ejemplo, la categoría «saludable/no saludable». Los discursos nutricionales, aún sin pretenderlo, muy a menudo acaban clasificando los alimentos y sus formas de consumirlos como más o menos aconsejables para la salud, y estos discursos son ampliamente difundidos, no solo por los profesionales sanitarios, sino, sobre todo, por 
los medios de comunicación. Por ello, podemos suponer que los contenidos de esta categoría serán objeto de cierto consenso. En efecto, en términos generales, algunos elementos de esta categoría parecen claros, al menos en primera instancia. Así, suele haber pocas dudas a la hora de incluir a frutas, verduras y hortalizas frescas en la categoría de "alimentos saludables». Son, de hecho, el paradigma de esta categoría. Pero cuando nos adentramos en las declinaciones de estas categorías generales de alimentos, empiezan a aparecer las discrepancias, y esta es una de las cuestiones que se aborda en el presente estudio. En definitiva, aunque los discursos sobre alimentación saludable — tanto los rigurosos y científicos como los que no lo son tanto- parecen ejercer una gran influencia en las categorizaciones, también ofrecen un amplio margen a las interpretaciones individuales. Y lo mismo puede decirse de otras categorías ampliamente utilizadas, como la de "alimentos engordantes/no engordantes». La permeabilidad de las categorías es, pues, un primer aspecto a analizar. Otro aspecto interesante es el de las influencias recíprocas entre sistemas de clasificación de los alimentos y comportamientos alimentarios. Parece poco discutible que nuestra forma de clasificar los alimentos incide en nuestras elecciones alimentarias. Pero también nuestras elecciones alimentarias inciden en nuestra forma de clasificar los alimentos. Así, los alimentos que consumimos a diario entraran en la categoría de "alimentos cotidianos", los que nos gustan en la categoría de "sabrosos» y los que no podemos consumir por escasos o excesivamente caros en la categoría de «inaccesibles o excepcionales». De este modo, los alimentos incluidos en una u otra de estas categorías también varían de un individuo a otro, en función de sus hábitos, de sus preferencias y de su poder adquisitivo.

Conviene asimismo destacar que, aunque sistemas de clasificación -como categorizamos los alimentos y comportamientos alimentarios, lo que comemos- se influyen recíprocamente, no se trata de relaciones simples y lineales, sino de interacciones complejas. Esto no resulta sorprendente, puesto que sabemos que en los comportamientos alimentarios inciden toda una serie de factores en interacción, genéticos y fisiológicos, pero también emocionales (Macht, 2008), cognitivos, psicosociales, sociales y culturales (Bellisle, 2009; Contreras, 1993; Köster, 2009; Rozin, 2002; Rozin y Vollmecke, 1986), y la categorización de los alimentos se construye también mediante la interacción de todos estos factores.

\section{Objetivos e hipótesis}

En el presente artículo se presentan los resultados de un estudio realizado los años 2014-15 y desarrollado en Aragón y en Cataluña sobre la cate- 
gorización de los alimentos. La pregunta inicial es: «¿Cuál es el peso de las categorías relativas a la construcción del cuerpo en las elecciones alimentarias?» Para tratar de responderla es preciso explorar las diferentes categorías a partir de las cuales se clasifican los alimentos en nuestra sociedad, identificar los consensos y las discrepancias que se producen entre los individuos a la hora de incluir los alimentos en una categoría u otra, abordar los desplazamientos de un mismo producto entre diferentes categorías y analizar el efecto de este desplazamiento en la valoración del producto. Exige identificar las categorías que son evocadas más a menudo en primera instancia y de manera generalizada y cuáles no aparecen hasta más tarde y no siempre. Partimos del supuesto de que las categorías que son evocadas en primer lugar son más accesibles, es decir, son utilizadas con mayor frecuencia que otras y que, por tanto, tienen un mayor peso en las elecciones alimentarias que otras que aparecen más tarde o que no son evocadas espontáneamente, salvo que se saquen a colación.

Se han planteado las hipótesis siguientes:

a) En nuestra sociedad, las categorías relacionadas con la construcción del cuerpo («saludable»/«no saludable»y «engordante»/«no engordante», son ampliamente utilizadas). Estas aparecerán con mayor frecuencia que otras categorías. Serán categorías muy accesibles.

b) Algunas categorías no surgirán en primera instancia, solo lo harán cuando se siga ahondando en el tema. Serán categorías poco accesibles.

c) Algunos productos se integrarán fácilmente y de manera altamente consensuada en determinadas categorías, mientras que otros generarán dudas y serán objeto de discusión.

d) Se producirán desplazamientos en la categorización de los alimentos entre la primera fase del estudio, en la que solo se evoca la idea de un alimento determinado (se lo nombra) y la segunda fase, en la que se entra en contacto con el alimento real (se toca, se ve, se huele).

\section{Metodología}

A continuación, se presentan las técnicas utilizadas para la recogida de datos y el procedimiento seguido en su aplicación.

\subsection{Presentación de las técnicas utilizadas}

Se ha optado por una combinación de tres técnicas cualitativas: la asociación de palabras, las tareas de clasificación y el grupo de discusión, com- 
binación que se ha evidenciado eficaz en la investigación en el ámbito de la alimentación (Guerrero, Claret, Verbeke, Enderli, Zarowska-Biemans, Vanhonacker, Issanchou, Sajdakowska, Granli, Scalvedi, Contel y Herleth, 2010; Guerrero, Claret, Verbeke, Vanhonacker, Enderli, Sulmont-Rossé, Hersleth y Guardia, 2012).

a) La asociación de palabras es una técnica utilizada para evaluar estructuras conceptuales (Hirsch y Tree, 2001; Hovardas y Korfiakis, 2006; Ross, 2003). Parte del presupuesto de que sí se proporciona un concepto estímulo y se pide que se asocie libremente con otras ideas que vengan a la mente; es posible acceder a representaciones relativas a ese concepto. Es una técnica rápida y que se ha demostrado efectiva en otros estudios (Ares, Giménez y Gámbaro, 2008; Guerrero et al., 2010; Roininen y Lahteenma, 2006; Rozin, 2002). Según la Teoría de la acción razonada, de Ajzen y Fishbein (1980), la mayor parte de las primeras asociaciones que los consumidores realizan sobre un determinado producto están estrechamente relacionadas con su conducta de compra. Se afirma incluso que las primeras asociaciones que se establecen pueden ser las más relevantes en las elecciones alimentarias (Roininen y Lahteenma, 2006). Benthin, Slovic, Moran, Severson, Mertz y Gerrard (1995) evidenciaron que la asociación de palabras es una técnica útil para detectar estas primeras asociaciones. Se ha argumentado que las ideas expresadas mediante libre asociación están sometidas a menores constricciones que las que imponen las entrevistas o los cuestionarios cerrados, lo que permitiría aflorar representaciones no previstas (Wagner, Valencia y Elejebarrieta, 1996). Sin embargo, la interpretación de los datos es compleja, por lo que se recomienda complementarlos con los obtenidos mediante otras técnicas y triangular los resultados.

b) Tarea de clasificación: es una técnica basada en el mecanismo cognitivo de la categorización, que permite abordar las diferencias y similitudes percibidas entre un conjunto de productos. Se ha utilizado con resultados interesantes en el estudio del concepto de alimentos tradicionales (Guerrero et al., 2012). Permite ver con qué frecuencia y en qué orden aparecen las categorías que se ha hipotetizado que emergerán y también que aparezcan categorías no previstas de antemano. No cierra ninguna posibilidad, ni orienta a los participantes hacia ninguna categoría concreta. Además, permite identificar qué representantes se eligen para cada categoría, si existe acuerdo entre los participantes, si se producen solapamientos o abiertas contradicciones y ver cómo los alimentos se desplazan de una categoría a otra. Es, pues, un camino de doble dirección, que lleva del producto a la categoría genérica y de la categoría genérica al producto. 
c) Grupo de discusión. Consiste en la discusión en grupo —entre cuatro y ocho personas- sobre una serie de cuestiones introducidas por el moderador, durante un período de tiempo determinado, que oscila entre los noventa minutos y las dos horas. Es una herramienta muy eficaz para abordar los criterios de asignación de los alimentos en una categoría u otra.

\subsection{Procedimiento}

Se han realizado diez sesiones, cinco en Barcelona y cinco en Teruel. En cada sesión han participado ente cuatro y seis personas, hombres y mujeres, de 18 a 55 años, con un nivel de formación medio-alto, residentes en zonas urbanas. Se ha considerado criterio de exclusión el tener alguna relación con la producción de alimentos o ser expertos en alimentación y nutrición. Esta muestra no tiene ninguna pretensión de representatividad y la elección se justifica más adelante. La duración de las sesiones ha oscilado entre los 150 y 180 minutos.

Cada sesión consta de diferentes partes. Al inicio de la sesión, se pide a cada participante que llene, de manera individual, un pequeño formulario, en el que se recogen los datos sociodemográficos básicos (sexo, edad, lugar de residencia, nivel de estudios) y se plantean las preguntas siguientes: "iTe ocupas habitualmente de la compra de alimentos en casa?"; "¿Cocinas habitualmente en casa?" "¿Solo para ti o para alguien más?»; "¿Sigues alguna dieta?» "En caso afirmativo ¿Cuál?»; "iSufres algún trastorno o enfermedad que afecte o se relacione con la alimentación?»

A continuación, se procede a explicar la primera tarea, la asociación libre de palabras. Cuando se ha asegurado la comprensión de la tarea, esta da comienzo. El orientador/a de la sesión dice en voz alta y de manera clara cada uno de los conceptos de una lista previamente establecida y deja tiempo para que cada participante escriba de manera individual y en silencio la primera palabra que le evoque ese concepto. Inmediatamente después de que acaben de escribir pasa a la palabra siguiente. Cada participante está concentrado en su tarea, sin intercambiar ningún comentario con el resto. La lista incluye los 25 conceptos siguientes: leche de soja, patata, puré de patata de sobre, bolsa de magdalenas, barra de pan, pan de molde, croissant, arroz integral, arroz precocinado, cereales de desayuno, manzana, muesli ecológico, queso de oveja, tomate triturado, lata de sardinas, queso en lonchas, jamón serrano en lonchas, garbanzos cocidos, lata de albóndigas, zanaboria, zumo de naranja, leche enriquecida con calcio, lata de fabada, tomate, bote de maíz en grano. Se han seleccionado los productos de la lista intentando que estuvieran representados produc- 
tos frescos y productos procesados en mayor o menor grado, productos que exijan elaboración y productos de preparación rápida, productos de consumo frecuente, productos de consumo ocasional y productos con alegaciones específicas (ecológicos, enriquecidos). Aunque se deja el tiempo suficiente para que todo el mundo pueda escribir, la respuesta debe ser rápida.

Cuando ha concluido, se explica a los participantes la tarea de clasificación, que consiste en clasificar los alimentos del listado en diferentes categorías. Se ponen ejemplos de otros temas (por ejemplo, deportes: «Si se tratara de deportes se podría clasificar en deportes de equipo y deportes individuales, deportes de agua o deportes de nieve, etc.») para que entiendan lo que se espera de ellos, pero sin proporcionar otros indicios que orienten las respuestas en una determinada dirección. No se propone ningún criterio de clasificación; se les anima a que piensen en estos criterios y que establezcan el número de categorías que les parezca oportuno. Esta tarea se realiza individualmente y en silencio y se da por finalizada cuando el último participante deja de escribir. Su duración ha oscilado entre 15 y 30 minutos.

A continuación, el orientador/a pone encima de la mesa todos los alimentos que aparecían en el listado y se inicia la parte grupal de la sesión. Se permite a los participantes tocar los alimentos y leer las etiquetas, y se les anima a moverse alrededor de la mesa y desplazar los productos sobre esta. Se les pide de nuevo que propongan categorías y que decidan entre todos qué alimentos incluir o no en estas, agrupando en distintos extremos de la mesa los que pertenecen a las diferentes categorías. Esta fase, que se graba en audio y vídeo, es la más larga y da lugar a debates de una gran riqueza.

Los términos aparecidos en la primera tarea, la de libre asociación, se han agrupado por proximidad semántica; primero, de manera independiente por los dos investigadores, y después se ha procedido a consensuar estas agrupaciones. Las categorías que han emergido en la segunda tarea, la de clasificación, se han ordenado en función del orden de su aparición en cada caso. Por ejemplo, la categoría «saludable» aparecía siempre, pero en algunos casos como primera categoría evocada, y en otros en segundo o tercer lugar, de manera que se han contabilizado cuántas veces aparecía cada categoría, pero también cuántas veces lo hacía en cada posición. Por lo que respecta a la tarea grupal, la grabación en audio se ha transcrito íntegramente y estas transcripciones han sido codificadas. La asignación definitiva de las unidades de texto a cada categoría de análisis se ha realizado mediante contraste entre los investigadores, para mejorar la fiabilidad del proceso. A continuación, se ha procedido al análisis de los resul- 
tados de la codificación. La grabación en vídeo se ha utilizado como apoyo para ilustrar cómo se van situando en cada sesión los diferentes productos, y su ubicación final en la mesa. Al tratarse solo de una herramienta de apoyo no se ha utilizado una plantilla de vaciado sistemático.

\subsection{Justificación de la orientación del estudio}

Desde el punto de vista del tema que nos ocupa, las ciencias cognitivas nos explican por qué y cómo los seres humanos abordamos el mundo estableciendo categorías. Se trata de un proceso no consciente y fundamental para la supervivencia. Pero sí es preciso explicar en cada caso los contenidos de los sistemas de clasificación, que son histórica y culturalmente contingentes. Las técnicas cualitativas propuestas permiten abordar esta tarea, lo que no excluye que otras técnicas puedan ser igualmente útiles y complementarias y que una aproximación cuantitativa pueda ser necesaria en fases posteriores. Los comportamientos alimentarios son un fenómeno complejo y, como señala Warde (2014), los fenómenos de investigación complejos exigen la intervención de múltiples métodos, tanto de recogida de datos como de análisis de estos.

Por lo que respecta al marco teórico dentro del que se analizan - $-\mathrm{y}$ se interpretan - y adquieren sentido los datos, resulta poco discutible que una comprensión cabal de los comportamientos alimentarios exige tener en cuenta todas las dimensiones que lo constituyen (fisiológicas y psicológicas, sociales y culturales), contemplar todas las variables intervinientes, y hacerlo no de manera aislada, abordando cada una de estas de manera independiente, sino en sus múltiples y complejas interacciones. Esto exige un modelo integrador y comprensivo que permita interpretar todos los datos y hacer predicciones fecundas. Pero, para que un modelo explicativo integrador sea potente, es preciso abordar las partes con la mayor precisión posible. Una de estas partes a analizar es el establecimiento de categorías alimentarias, de complejos sistemas de clasificación, que influyen y son influidos por las elecciones y el comportamiento alimentario mismo.

El presente estudio se centra exclusivamente en las categorizaciones, sin abordar el medio cultural del que emergen, sin contemplar los mecanismos psicológicos subyacentes y sin analizar cómo y en qué medida sistemas de clasificación y comportamientos se relacionan. Es decir, se focaliza en un ámbito estrecho del hecho alimentario. Esto no quiere decir que se considere que los sistemas de clasificación se generen fuera de un entramado sociocultural concreto, ni, por supuesto, que este sea irrelevante. Muy al contrario, es absolutamente esencial. El hecho de poner el foco 
en las categorizaciones no supone considerar que estas se producen en el vacío. Se trata simplemente de centrar la atención en un determinado fenómeno, para contribuir, en alguna medida, a su comprensión. Pongamos por ejemplo el caso de las omnipresentes consideraciones, en nuestra sociedad, sobre peso e imagen corporal. Parece poco discutible que estas tienen importancia en nuestras actitudes hacia los alimentos. Ahora bien, ¿es posible inferir por ello que estas consideraciones determinan nuestros sistemas de clasificación y que lo hacen siempre, para todos, de la misma manera, en la misma dirección? El denso entramado sociocultural en el que se llevan a cabo las categorizaciones es ineludible. Las categorías establecidas por nuestros informantes solo tienen sentido en una sociedad en la que la mayor parte de la población compra los alimentos en lugar de producirlos, dentro de un determinado sistema de producción, transformación, conservación y distribución de los productos, y bajo el influjo de formas concretas de puesta en valor de estos (publicidad, estrategias de márquetin, discursos médicos, saber científico, etc.). Nuestra pregunta no es si el universo cultural en el que las categorizaciones se formulan y se despliegan es relevante - la respuesta es rotundamente afirmativasino ver cuáles son las categorizaciones concretas que este genera. Se trata de una perspectiva limitada, voluntariamente reduccionista, pero que parte de la consideración de que la integración de este tipo de aportaciones muy focalizadas puede contribuir a una mejor comprensión de la alimentación como la manifestación cultural total que es.

\section{Resultados}

Como se ha expuesto anteriormente, se han realizado tres aproximaciones sucesivas a los productos y a su categorización: 1) asociación de palabras, 2) establecimiento individual de categorías y 3) discusión grupal con los productos físicamente presentes. La atención se ha focalizado en los patrones de clasificación que han emergido a lo largo de todo el proceso: por un lado, comparando los datos obtenidos del conjunto de participantes en cada paso y, por el otro, abordando las respuestas de un mismo individuo en las tres tareas. Por lo que respecta la asociación de palabras, y analizando los datos globales, lo primero a destacar es que, a pesar de la diversidad de términos evocados, algunas asociaciones son recurrentes y esto sucede con todas las palabras de la lista. El agrupamiento de los términos evocados en categorías con una etiqueta ha sido establecido por los investigadores, a efectos analíticos, pero intentando respetar al máximo su cercanía (por ejemplo, en el grupo «momento de consumo» se han incluido «desayuno», "comida», «merienda», «cena», «almuerzo», "sá- 
bado» o «fin de semana»). A continuación, se han ordenado estas categorías en función del número de veces que aparecían los términos incluidos en cada una de ellas. Las categorías ordenadas por frecuencia de aparición son: a) «salud, y efectos sobre el organismo», b) «forma de consumo», c) «características organolépticas», d) «momento de consumo», e) «conveniencia», f) «naturalidad/artificialidad», g) «lugar de consumo», h) «marca comercial», i) «origen». En algunos casos el término evocado era un nombre propio (Jorge, María...), o relativo a un miembro de la familia (papá, mamá, abuela...). Ocasionalmente, se ha asociado el producto con el precio ("caro", "barato») y solo en una ocasión se ha asociado un producto de la lista (puré de patas envasado) con toxicidad, en un caso con "poco seguro" (arroz precocinado) y, en otro, el término maíz en grano envasado se ha asociado con «transgénico».

La categoría etiquetada como "salud y efectos sobre el organismo" incluye todos los términos relativos a la salud de manera directa ("sano», «saludable», «salud», «insano», «no saludable»), los relacionados con el peso («dieta», «engorda», "peso») y los relativos a efectos sobre el organismo ("visión», «huesos», «menopausia», «embarazadas», «flatulencia», «hinchazón», «gases», «digestión», «fibra», «energía»). Algunos términos son problemáticos, como por ejemplo «graso» y "grasiento", ya que pueden referirse a la salud, a la imagen corporal o a los efectos sobre el organismo, y por lo tanto pueden integrarse en esta categoría, o pueden referirse a características organolépticas, y pertenecer a otra. En estos casos se ha decidido su inclusión en una determinada categoría en función de las respuestas dadas por los mismos participantes en la tarea de clasificación. Las palabras relacionadas con esta primera categoría son las más citadas, representando el $23 \%$ de las asociaciones libres. Además de integrarse en una u otra categoría, como se ha dicho anteriormente, los términos pueden tener una valencia positiva y negativa. Las palabras que se han asociado con mayor frecuencia con la construcción del cuerpo en términos positivos son, por este orden: leche enriquecida con calcio, leche de soja, zanahoria, arroz integral, muesli ecológico, cereales de desayuno y manzana. Los garbanzos de producción ecológica, el zumo de naranja envasado y el tomate fresco se asocian con «sano" o «saludable» el mismo número de veces (cuatro respectivamente, frente a 22 veces la leche enriquecida con calcio). Las palabras que se han asociado con mayor frecuencia con términos negativos relativos a la construcción del cuerpo han sido, por este orden: fabada enlatada, albóndigas enlatadas, lata de sardinas, magdalenas y croissant. El número de términos negativos relativos a la construcción del cuerpo es muy inferior al de términos positivos $(6 \%$ y $20 \%$, respectivamente). Respecto a las palabras asociadas a la forma de 
consumo (formas de preparación y platos), representan el $21 \%$ de las asociaciones. Destacan, por este orden, las asociaciones siguientes: queso en lonchas (sándwich o bocadillo), pan de molde (sándwich o bocadillo), patata (fritas, asadas, tortilla, guarnición), maíz en grano (ensalada), tomate (ensalada) y zanahoria (ensalada). Las asociaciones relacionadas con características organolépticas («sabroso», «bueno», «asqueroso», «malo», «mal gusto», «crujiente», «blando», «seco», «dulce», «insípido», «pegajoso"...) representan el $20 \%$ del total. El mayor número de asociaciones positivas se relacionan con: croissant, magdalenas envasadas, cereales de desayuno y muesli ecológico. Las negativas se concentran sobre todo en: albóndigas enlatadas, arroz precocinado, sardinas enlatadas y, con menor frecuencia, zumo de naranja envasado. Algunos productos evocan el mismo número de asociaciones negativas y positivas: queso idiazábal, jamón envasado y garbanzos de producción ecológica. Los términos relacionados con el momento de consumo («desayuno», "comida», «merienda», «cena», «fin de semana», «diario»...) representan el 10\% de las asociaciones. Los productos que más asociaciones han generado en este sentido son: magdalenas envasadas, croissant y zumo de naranja (16, 13 y 12 respectivamente, asociadas todas a «desayuno»). Bajo la etiqueta de «conveniencia» ("prisa», «fácil», «rápido», «salvación», "comodidad»...) se agrupa el $6 \%$ de las asociaciones, relacionadas la mayor parte de veces con el arroz precocinado, y con mucha menor frecuencia, con el puré de patatas envasado y las sardinas enlatadas. Los términos "artificial», "procesado», «industrial»y «natural» representan también el 6\% de las asociaciones. Hay una serie de términos problemáticos, como "campo", «huerto» o "pueblo», de discutible adscripción a esta categoría. Se ha decidido su inclusión o no, caso por caso, en función de los resultados de la tarea de clasificación del mismo participante. El puré de patatas es el que más se asocia a «artificial», "procesado» e «industrial», seguido de zumo de naranja envasado, albóndigas enlatadas, fabada enlatada y leche enriquecida con calcio. Los que se asocian en mayor medida con el término «natural» son: patata, zanahoria, tomate fresco y queso idiazábal, y con menor frecuencia garbanzos ecológicos. La leche de soja se asocia cinco veces con «natural» y cinco veces con «artificial». Debe señalarse que queso en lonchas, sardinas enlatadas, tomate triturado, maiz envasado y cereales de desayuno, todos ellos productos industriales, no se asocian en ningún caso con términos de esta dimensión. "Lugar de consumo» ( «hospital», «residencia», «camping», «campamento»), «marca comercial» y «origen» generan alrededor del $2 \%$ respectivamente de asociaciones. Se relacionan con «marca comercial» solo determinados productos industriales (puré de patatas, magdalenas envasadas y fabada) y el «ori- 
gen» se concentra en un número muy reducido de productos (queso idiazábal y fabada).

En la segunda tarea, la de clasificación, las categorías utilizadas por los participantes con mayor frecuencia son también las relacionadas con la construcción del cuerpo: "saludable», «no saludable», "para dieta», «energéticos», «calóricos», siendo «saludable»/«no saludable» las más frecuentes, seguidas de «dieta»/«para adelgazar»/«para mantener el peso»/«calóricos». La segunda categoría más citada es la que se podría etiquetar como «artificial»/«natural», aunque los participantes utilizan además de estos, otros términos: «industriales», "procesados», "precocinados», «envasados», «conservas». Se ha decidido si categorías como «conservas» o "envasados» se podían equiparar a "artificial» a partir de los criterios explicitados por los mismos participantes en el grupo de discusión. En tercer lugar, aparece la categoría relativa a las características organolépticas: «gustan», «no gustan», «sabrosos», «malos», «dulces», «salados». Estas tres categorías han aparecido prácticamente siempre, pero mientras que la relativa a la construcción del cuerpo y la relativa al grado de procesamiento pueden aparecer indistintamente en primer o segundo lugar, la relativa al sabor nunca es la primera en utilizarse. La siguiente categoría, por orden de frecuencia, es la relativa al momento de consumo: "desayuno", "cena», etc., seguida por la relativa a la familiaridad con el producto: "de consumo/compra frecuente», "de consumo/ compra ocasional», "que nunca se compra/consume». Aunque estas dos categorías aparecen muy a menudo, no están nunca en primer o segundo lugar. Otra categoría bastante citada, pero en menor medida, es la de "productos ecológicos». Ahora bien, es posible que la inclusión de productos explícitamente etiquetados como «ecológicos» haya sugerido esta categoría. Finalmente, otras categorías han sido citadas de manera ocasional, como las relativas a grupos de alimentos o macronutrientes, la de «animal»/«vegetal», las relativas al precio («caros»/«baratos») o las relativas a la conveniencia, («fáciles»/ «rápidos de preparar/consumir»).

En la tercera parte, la grupal, las categorías relativas a la construcción del cuerpo y a la artificialidad/naturalidad han sido sistemáticamente las primeras en aparecer. No se puede señalar la ausencia de alguna de estas dos categorías en ninguno de los grupos. Se trata también de las categorías a las que se ha dedicado más tiempo y han generado más discusión, cuando se pedía que distribuyeran los productos en la mesa en función de su inclusión en una categoría u otra. Los productos vegetales no procesados (tomate, patata, zanahoria, manzana) se han incluido de manera generalizada y sin generar dudas en la categoría de «saludables», y las albóndigas en lata en la de "no saludables». Pero el resto de productos han sido 
objeto de discusión dentro de cada grupo y han sido clasificados de forma distinta en las diferentes sesiones.

La mayor parte de veces la distribución resultante no consistía en varios grupos de alimentos netamente separados, sino un continuo graduado de menos a más en función del criterio de elección. Por ejemplo, si el criterio era "saludable/no saludable», al final sobre la mesa había un pequeño grupo de alimentos en un extremo (los «más» saludables), otro pequeño grupo en otro extremo (los «menos» saludables), y entre unos y otros una sucesión de productos sin solución de continuidad. Más importante aún es destacar que la colocación de los alimentos en este orden la mayor parte de veces era objeto de discusión, que iba desde las matizaciones hasta el abierto desacuerdo. El ejemplo más claro en este sentido es el zumo de naranja envasado. Una parte de los participantes lo situaba justo a continuación de los «saludables» por excelencia (fruta y hortalizas frescas), mientras que otros lo situaban casi en el extremo opuesto, aunque antes que las albóndigas o la fabada, paradigma de la categoría «no saludable». Y entre uno y otro extremo, los distintos participantes, y en los distintos grupos, lo iban moviendo en una u otra dirección, ocupando puestos ligeramente distintos en la ordenación final, expresando muchas dudas y presentando argumentos diversos. En términos generales, los que situaban el zumo de naranja en el lado "saludable» de la mesa aducían que se trataba de fruta y la fruta es saludable —es decir, lo categorizaban en primer lugar como «fruta»-, mientras que los que lo situaban en el extremo opuesto, argumentaban que se trataba de un producto artificial, lo categorizaban en primer lugar como «producto industrial». Un aspecto importante de esta última parte grupal consistía en que los participantes pudieran interactuar físicamente con los productos, a diferencia de las fases anteriores en las que solo se los nombraba. Este cambio suponía poco o ningún cambio a la hora de categorizar los productos frescos, pero sí introducía elementos nuevos cuando se trataba de productos industriales. Estos aparecían a raíz de consultar las etiquetas y de valorar la información que estas proporcionan. Un producto categorizado antes como «saludable» (como zumo de naranja), o al que no se había categorizado desde esta perspectiva (como maíz en grano), se desplazaban cuando se leían los ingredientes. Esto se hizo particularmente patente con los cereales de desayuno, que pasaron de ser considerados «muy saludables» al principio de la sesión, a situarse prácticamente al otro extremo después de mirar con atención su composición y de comentarlo entre los participantes. En el caso de la patata se producía un desplazamiento en sentido opuesto. En la asociación de palabras muchos la relacionaron con «fritas», por lo que, en la segunda tarea, la incluyeron en la categoría de «no salu- 
dables» o "engordantes», pero al ver la patata cruda encima de la mesa la situaron sin dudar en el grupo de productos más saludables.

\section{Discusión}

Se habían planteado inicialmente cuatro hipótesis, que se van a discutir a la luz de los resultados. Según la primera, las categorías «saludable»/«no saludable»y «engordante»/«no engordante», muy presentes en nuestra sociedad, deberían aparecer en primera instancia y con mayor frecuencia que otras categorías, indicando que se trata de categorías muy accesibles. Esta hipótesis se confirma, tanto en la primera como en la segunda tarea, aunque debe destacarse que, junto a estas dos, emerge también la categoría "forma de consumo», que parece ser clave a la hora de pensar un determinado alimento. Si a esta se le añade "momento de consumo", su peso es muy destacable, superando a la categoría relativa a la construcción del cuerpo. Ambas categorías, "forma de consumo» y «momento de consumo», pueden considerarse relacionadas con familiaridad con el producto evocado. Cuando se trata de alimentos, y de un acto cotidiano como el de comer, la familiaridad con el producto es determinante. Por ejemplo, solo uno de nuestros informantes ha calificado de manera positiva, en las dos primeras tareas, la lata de albóndigas. En la asociación libre la relaciona con «rico», y en la tarea individual de clasificación la incluye en las categorías de «conveniencia», por su facilidad de preparación, y de «preferencia» (categoría «alimentos que gustan»). En la tarea grupal tiene más dudas que el resto a la hora de situarla en la parte de la mesa destinada a alimentos no saludables, aunque no cuando se trata de clasificarla en las dimensiones «artificial»/«natural». Y es también la única persona que afirma consumir este producto con cierta frecuencia. Esto sugiere que la percepción que se tiene de un producto es subsidiaria, en gran medida, de que se esté familiarizado con él o no. En la misma dirección, los participantes que afirmaban estar, en la discusión grupal, muy familiarizados con la leche enriquecida con calcio - por tomarla ellos mismos o sus madres o abuelas- la percibían no solo más saludable, sino también menos industrial, más natural que el resto. Esto se relaciona con otro aspecto que se ha observado en la parte grupal: los participantes que han relacionado un determinado alimento con «saludabilidad», tanto en la tarea de asociación libre como en la de categorización, tienden a situarlo, en la tarea grupal, más cerca del grupo de alimentos «naturales» que de los «artificiales», aunque se trate de productos industriales, como la leche de soja o el tomate triturado enlatado. Es decir, parece que se produce un solapamiento entre el concepto de «saludable» y el de «natural», que hace los productos indus- 
triales etiquetados como «buenos para la salud» sean percibidos también como más "naturales", solapamiento que parece producirse también entre los alimentos etiquetados como «buenos para la salud», $\mathrm{y}$ «buenos para el control del peso", y que produce desplazamientos en las dos direcciones: los alimentos que se han asociado con beneficios para la salud se etiquetan a su vez como buenos para el control del peso, y viceversa, aunque tal relación sea inconsistente. Así, los participantes que han asociado el arroz integral con beneficios para la salud, también lo categorizan como «beneficioso para el control del peso» y como «natural», Y esto, independientemente del grado de procesamiento del producto.

La segunda hipótesis predecía que algunas categorías aparecerían sistemáticamente en los primeros lugares, mientras que otras solo surgirían cuando se siguiera ahondando en el tema. Como se acaba de explicar, esta hipótesis se ha confirmado. Es en este sentido que se puede hablar de categorías ausentes, como las relativas a la seguridad/toxicidad de los alimentos, o sus dimensiones medioambientales o éticas. Su ausencia, o su presencia muy marginal, pueden interpretarse como baja accesibilidad. Dicho de otra manera, estas categorías tendrían poco peso en las elecciones alimentarias. Que la dimensión medioambiental de los alimentos no es clave en las elecciones de la mayor parte de la población es sabido, a pesar de los discursos crecientes en nuestro entorno al respecto. Pero que la «seguridad alimentaria» que, por un lado, es clave para la supervivencia, y que, por el otro, tiene un largo recorrido mediático, sea una categoría ausente puede parecer más sorprendente. O no, si se tiene en cuenta que los alimentos que resultan familiares se perciben como seguros. Quizás sea algo más que anecdótico que en los dos casos en que un producto se ha asociado con «toxicidad» o «riesgo potencial» (arroz precocinado y maíz en grano) en la sesión grupal, las dos personas implicadas han afirmado no haberlo comido nunca. En cualquier caso, esto plantea la cuestión del sesgo que puede generar en las encuestas, entrevistas o grupos de discusión, el hecho de focalizar la atención en determinadas dimensiones como la seguridad alimentaria, el origen o cuestiones medioambientales. Se puede estar sobrevalorando el peso de estas consideraciones en las elecciones alimentarias.

Por lo que respecta a la tercera hipótesis, que propone que algunos productos se integrarían fácilmente y de manera altamente consensuada en determinadas categorías, mientras que otros generarían dudas y serían objeto de discusión, se ha confirmado plenamente. Como se esperaba, solo algunos productos se integran de manera casi unánime en alguna categoría: con valencia positiva, fruta y hortalizas frescas se integran sin discusión tanto en la categoría relativa a la «salud», como en la relativa a la «natu- 
ralidad», y los mismo sucede, con valencia negativa, con albóndigas enlatadas, fabada y magdalenas envasadas. La categoría "artificial/industrial» parece particularmente interesante en este sentido. Nuestros datos apuntan a que los criterios que se utilizan para incluir un alimento o no en esta categoría son sumamente imprecisos y fluidos. El hecho de que un producto sea procesado no es relevante. El queso, el pan o el jamón son alimentos procesados, pero no son percibidos, la mayor parte de veces, como «artificiales» o «industriales». La forma de presentación del producto parece ser más influyente, ya que se observa una tendencia a situar en un punto más alejado de la naturalidad los productos envasados. Pero no es un criterio estricto: el queso en lonchas es un producto industrial envasado, pero nadie, en la asociación libre, lo asocia con «artificial». Sí que parece que los productos enlatados se perciben más industriales que el resto, pero tampoco es determinante: la lata de tomate triturado es percibida a menudo como «bastante natural». Un caso interesante es el de la leche de soja de producción ecológica, categorizada por unos como «natural», y por otros como «artificial». ¿Cuál de sus características determina que se sitúe en una categoría u otra? La etiqueta de «ecológica» no la hace más natural para algunos, por lo que la clave puede residir en su asociación con la salud. Son aquellos que, en la asociación de palabras, la han relacionado de alguna manera con «saludabilidad», los que han tendido luego a considerarla más natural. Pero el elemento que más asociaciones positivas ha suscitado, desde la perspectiva de la salud, la leche enriquecida con calcio, también se ha asociado en cinco casos con «artificial»y «antinatural». Es esta una cuestión que conviene seguir explorando.

La cuarta hipótesis predecía que se producirían desplazamientos en la categorización de los alimentos entre la primera fase del estudio, en la que solo se evoca la idea de un alimento determinado (se lo nombra), y la segunda fase, en la que se entra en contacto con el alimento real (se toca, se ve, se huele). Esta hipótesis también se ha confirmado, como se ha señalado anteriormente. Se ha visto que un mismo producto se integra en diferentes categorías, con valencia positiva en unas y negativa en otras, $\mathrm{y}$ que no todas las categorías son igual de accesibles. Por mucho que en la parte grupal se situaran el croissant y las magdalenas industriales en el extremo de "poco saludable» y "poco adecuadas para el control del peso» (categorías relativas a la construcción del cuerpo), el hecho de que en la asociación de palabras evocaran términos positivos («bueno», «rico»), y, sobre todo, que se relacionaran con formas de consumo ("con leche», "con chocolate») y momentos de consumo ("desayuno», "merienda", «domingo») nos informa mucho más de la saliencia de cada categoría, y, por ello, de las elecciones alimentarias de los participantes. 


\section{Limitaciones}

Las limitaciones identificadas son de distinto orden, y se refieren principalmente a la muestra y al listado de alimentos utilizados. Respecto al número de participantes, su reducido número no permite explorar el papel de variables que pueden ser relevantes. En el grupo en el que participaban mujeres entre 30 y 50 años, la categoría relacionada con el control del peso aparecía antes que en los grupos compuestos por personas más jóvenes, tanto en la asociación libre de palabras como en la tarea de clasificación. También se ha observado que los informantes que practicaban regularmente deporte clasificaban a menudo los alimentos en función de sus efectos sobre el rendimiento deportivo, mientras que el resto de participantes no lo hacía. Asimismo, en relación con las preguntas iniciales relativas a la responsabilidad o al seguimiento de dietas hay indicios de relaciones entre las respuestas a estas y patrones ligeramente diferentes de clasificación. Replicar el estudio con un número de participantes mayor, permitiría discriminar en función de variables clave, como la edad, el sexo o el lugar de residencia, pero también de otras variables, como la práctica regular de ejercicio, el seguimiento de dietas o la responsabilidad en las tareas relacionadas con la alimentación, y ver si estos indicios se concretan en relaciones robustas.

En relación con las características de la muestra es preciso señalar que la mayor parte de los jóvenes son universitarios, y casi dos terceras partes de los adultos tienen también estudios superiores. Este sesgo en la muestra sin duda afecta los resultados, por lo que es deseable replicar el estudio con una muestra más representativa del conjunto de la población. Respecto al listado de productos, debe ser mejorado en futuros trabajos, ya que en algunos casos genera confusiones o induce categorías y sería deseable introducir un mayor número de productos no procesados, que no fueran vegetales, para ver si la relación entre fresco y saludable es constante o varían en función de que se trate de un producto fresco vegetal o animal.

\section{Para finalizar}

A pesar de las limitaciones citadas, se considera que las técnicas utilizadas son útiles para abordar la cuestión de las categorizaciones alimentarias. Los resultados de la asociación de palabras son consistentes con la tarea de clasificación individual, pero, al mismo tiempo, introducen elementos nuevos de contraste. Y la tarea de clasificación grupal, junto con la discusión posterior, contribuye a la interpretación de los resultados de la técnica anteriores. 
En respuesta a la pregunta inicial: "¿Qué peso tienen las categorías relacionadas con la construcción del cuerpo en nuestras elecciones alimentarias?", es posible afirmar que estas parecen ser relevantes en las elecciones, dada su aparente gran accesibilidad. Pero esta afirmación debe matizarse a la luz del peso que parece tener la familiaridad con el alimento en cuestión para su inclusión en una u otra categoría. Se ha visto que una de las maneras de pensar un alimento tiene que ver con cuándo, cómo, y con quién se consume. Los alimentos que se consumen habitualmente, y muy en particular en un contexto que inspire confianza, como es el familiar, no solo no son percibidos como dañinos para el cuerpo, sino que se perciben además como más "naturales». Asimismo, se observa la clara tendencia a clasificar en función de su supuesta «naturalidad». Ahora bien, cuáles son los criterios que determinan que un alimento sea «natural» no resulta tan evidente. Parece ser que la percepción de «naturalidad» o "artificialidad» depende de toda una serie de claves, que son, a su vez, susceptibles de interpretación individual. ¿Qué hace que la leche enriquecida con calcio sea percibida como "natural»? $¿$ ¿Las asociaciones positivas con la salud? ¿El hecho de que la madre o la abuela la consuman? ¿O que se trata de un producto con el que se está muy familiarizado? ¿Influye el hecho de que sea leche, y que esta se asocia con naturalidad, independientemente del grado de procesamiento al que haya sido sometida?

Nuestros resultados sugieren que, en nuestra sociedad, las categorías con las que clasificamos los alimentos se relacionan estrechamente, y por diferentes vías, con la construcción del cuerpo. Pero junto con las respuestas obtenidas, necesariamente parciales, se plantean sobre todo nuevas preguntas: ¿En qué medida incluir un alimento en una determinada categoría (por ejemplo, «saludable») influye en que sea incluido o no en otra (por ejemplo, «natural»)?, ¿cómo se construyen los contenidos de las categorías?, ¿en función de que variables?, ¿qué criterios se utilizan?, ¿qué papel tienen la familiaridad y los hábitos y preferencias aprendidas? Intentar responder a estas preguntas puede ser el punto de partida de futuros trabajos.

\section{Referencias}

Ajzen, I. y Fishbein, M. (1980). Understanding attitudes and predicting behavior. Engelwood Cliffs, NJ: Prentice-Hall Inc.

Ares, G.; Giménez, A. y Gámbaro, A. (2008). Understanding consumers perception of conventional and functional yogurts using word association and hard laddering. Food Quality and Preference, 19(7): 636-643. 
Basulto, J. y Cáceres, J. (2014). Comer y correr. desmontando los mitos de la alimentación. Barcelona: DeBolsillo.

Basulto, J. y Cáceres, J. (2016). Más vegetales, menos animales: una alimentación más saludable y sostenible. Barcelona: DeBolsillo.

Bellisle, F. (2009). How and why should we study ingestive behaviors in humans? Food Quality and Preference, 20: 539-544.

Benthin, A.; Slovic, P.; Moran, P.; Severson, H.; Mertz, C.K. y Gerrard, M. (1995). Adolescent health-threatening and healthenhancing behaviors: a study of word association and imagery. Journal of Adolescent Health, 17: 143-152.

Bredahl, L.; Grunert, K.G. y Frewer, L.J. (1998). Consumers attitudes and decision-making with regard to genetically engineered food products. A review of the literature and a presentation of models for future research. Journal of Consumer Policy, 21: 251-277.

Contreras, J. (1993). Antropología de la alimentación. Madrid: Eudema Universidad.

Contreras, J. (2005). La modernidad alimentaria. Entre la sobreabundancia y la inseguridad. Revista Internacional de Sociología, 40: 109-132.

Costa Font, M.; Gil; Trail, J.M. y Bruce, W. (2008). Consumer acceptance, valuation of and attitudes towards genetically modified food: Review and implications for food policy. Food Policy, 33(2): 99-111.

Gracia-Arnaiz, M. (2010). Fat bodies and thin bodies. Cultural, biomedical andmarket discourses on obesity. Appetite, 55: 219-225.

Guerrero, L.; Claret, A.; Verbeke, W.; Enderli, G.; Zarowska-Biemans, S.; Vanhonacker, F.; Issanchou, S.; Sajdakowska, M.; Granli, B.S.; Scalvedi, L.; Contel, M. y Herleth, M. (2010). Perception of traditional food products in six Europeans regions using free word association. Food quality and preference, 21: 225-233.

Guerrero, L.; Claret, A.; Verbeke, W.; Vanhonacker, F.; Enderli, G.; Sulmont-Rossé, C.; Hersleth, M. y Guardia, M.D. (2012). Cross-cultural conceptualization of the words Traditional and Innovation in a food context by means of sorting task and hedonic evaluation. Food Quality and Preference, 25: 69-78.

Hovardas, T. y Korfiatis, K.J. (2006). Word associations as a tool for assessing conceptual change in science education. Learning and Instruction, 16: 416-432.

Köster, E.P. (2009). Diversity in the determinants of food choice: A psychological perspective. Food Quality and Preference, 20, 70-82.

Lusk, J.; Jamal, M.; Kurlander, L.; Roucan, M. y Taulman, L. (2005). A meta analysis of genetically modified valuation studies. Journal of Agricultural and Resources Economics, 30: 28-44.

Macht, M. (2008). How emotions affect eating: A five-way model. Appetite, 50: 1-11.

Robertson, A.; Lobstein, T. y Knai, C. (2007). Obesity and socioeconomic groups in Europe: Evidence review and implications for action. En http://ec.europa.eu/health/ph_determinants/life_style/nutrition/documents/ev20081028_rep_en.pdf. Accedido el 4 de junio de 2017.

Roininen K. y Lahteenma, L. (2006). Exploring consumers perceptions of local food with two different qualitative techniques: Laddering and word association. Food Quality and Preference, 17: 20-30. 
Ross, T.P. (2003). The reliability of cluster and switch scores for the controlled oral word association test. Archives of Clinical Neuropsychology, 18(2): 153-164.

Rozin, P. (2002). Human food intake and choice: Biological, Psychosocial and Cultural Perspectives. En Food Selection. From Genes to Culture. H. Anderson, J. Blundell y M. Chiva, Eds. Paris: Danone Institute.

Rozin, P. y Vollmecke, T.A. (1986). Food likes and dislikes. Annual Review of Nutrition, 6: 433-456.

Wagner, W.; Valencia, J. y Elejabarrieta, F. (1996). Relevance, discourse and the hot stable core of social representations and structural analysis of word associations. British Journal of Social Psychology, 35: 331-351.

Warde, A. (2014). Food studies and the integration of multiples methods. Politica y Sociedad, 51: $51-72$. 
